\title{
A Social Network Recommendation Algorithm Based on Information Aging
}

\author{
Zhiyong Yang ${ }^{* 1,2}$, Haiyang Wang ${ }^{1}$, Shun $\mathrm{Li}^{2}$ \\ ${ }^{* 1}$ Department of Information Engineering, Chongqing Vocational Institute of Engineering, Chongqing, P.R China, 402260 \\ E-mail: zy.yang@cqu.edu.cn. \\ *2College of Automation, Chongqing University, Chongqing, P. R. China, 400044 \\ corresponding author : Zhiyong Yang, Email:yzy023@aliyun.com
}

\begin{abstract}
Aiming at the problem of low timeliness in social network recommendation systems, a new social network recommendation algorithm using information aging was presented in this paper. The paper proposed a trust network recommendation system based on trust mechanism and a timeliness evaluation model was established to predict the influence of the current time of information with rating records. A novel algorithm to find the current user's nearest neighbors in proposed trust network by the influence of the current information and a highly timeliness recommendation list was generated by the information of nearest neighbors. The practical results show that the proposed algorithm improves the timeliness of the recommendation list by comparing with the traditional recommendation algorithm. (Abstract)
\end{abstract}

Keywords: Social network; Information aging; Timeliness; Trust network

\section{INTRODUCTION}

With the rapid development of Internet, the amount of the social information is keeping increase. It is difficult for users to find the valuable information which attracts them in large-scale data [1]. Personalized recommendation enables users to obtain useful information more easily, and it has been widely used in many fields [2]. Personalized service is to take the initiative to meet user's personalized needs starting from the user's historical behavior. Collaborative filtering recommendation algorithm is the most widely used recommendation algorithm in the recommendation system. Collaborative filtering algorithm generates the recommendation by the historical behavior of similar users. However, along with the expansion of the data

Permission to make digital or hard copies of all or part of this work for personal or classroom use is granted without fee provided that copies are not made or distributed for profit or commercial advantage and that copies bear this notice and the full citation on the first page. To copy otherwise, to republish, to post on servers or to redistribute to lists, requires prior specific permission and/or a fee.

Mobimedia 2017, July 13-14, Chongqing, People's Republic of China

Copyright (๑) 2017 EAI 978-1-63190-156-0 interested in the content of the news because journalism is unique to timeliness. These novel recommendation algorithms mainly consider the rating and the trust to produce the recommendation for the current user. But the timeliness of some items in the recommendation list is relatively low, which will reduce the accuracy rate of the recommendation system.

There are few studies about information expiration in recommendation systems, but the time attribute of information has been applied in the research of recommendation system. Literature [5-7] believes that if the evaluation information is far from current time, the contribution of the information will not be effective in recommendation systems. These algorithms used attenuation function to describe the decay process of the information, but they do not consider that the failure rate of different resources is obviously different. A collaborative filtering recommendation model based on forgetting curve is proposed [8], which reveals the changing trends of the rate of forgetting about the currency of information. These studies mainly consider the timeliness of the user's preference behavior without considering the timeliness of the information itself. Literature [1] generates a preliminary recommendation list for current user by collaborative filtering algorithm, and then uses the information aging theory and click-through to filter the candidates to acquire a high timeliness information. Although the algorithm considers the timeliness of the information itself, the accuracy of the preliminary recommendation list is very low because of the sparsity of raw data.

This paper proposes a social network recommendation algorithm based on information aging to solve the problem of overdue information in the social network recommendation system. This paper establishes a social trust network based on trust mechanism [9], and the network uses rating records to establish timeliness evaluation model of information top, thus the influence of the current information is predicted. A novel algorithm to find the current user's nearest neighbors in proposed trust network by the influence of the current information and a highly timeliness recommendation list was generated by the information of nearest neighbors. The second section of the paper presents an improved information timeliness model [1], and uses the scoring records to predict the impact of the current information. In the third section, a social network algorithm based on 
information aging is proposed. In the fourth section, the experiment is carried out by using the public dataset and the experimental results are analyzed. The fifth section gives the conclusion.

\section{INFORMATION AGING THEORY}

Based on the algorithm of literature [1], the paper presents three concepts for the life cycle, the half life and the information influence. The definition of them is as follows.

Definition 1(Life Cycle $T_{\text {all }}$ ): The life cycle of information refers to the time period $\left(T_{\text {all }}\right)$ between the time when the information is released $\left(T_{\text {publish }}\right)$ and the time when the information is no longer being evaluated $\left(T_{\text {publish }}+T_{\text {all }}\right)$. Information has a certain influence in the life cycle.

Definition 2 (Half Life $T_{\text {half }}$ ): The half life of information refers to the time period $\left(T_{\text {half }}\right)$ between the release of information ( $\left.T_{\text {publish }}\right)$ and the time when the influence of the information goes down to half $\left(T_{\text {publish }}+T_{\text {half }}\right)$.

Definition 3 (Information Influence Influence $\left(T_{\text {current }}, T_{\text {publish }}\right)$ ): The influence of information is the ratio between the number of scores in the time period ( $\operatorname{Count}\left(T_{\text {current }}, T_{\text {publish }}\right)$ ) and the number of the entire life cycle ( $\operatorname{Count}\left(T_{\text {all }}\right)$ ), specific as shown in the formula (1).

$$
\begin{aligned}
& \text { Influence }\left(T_{\text {current }}, T_{\text {publish }}\right)= \\
& \left(\operatorname{Count}\left(T_{\text {current }}, T_{\text {publish }}+T_{\text {all }}\right) / \operatorname{Count}\left(T_{\text {all }}\right)\right) \times 100 \%
\end{aligned}
$$

Where, $T_{\text {current }}$ represents the current time, $T_{\text {publish }}$ represents the time of release of information, $T_{\text {all }}$ represents the life cycle of information, Count $\left(T_{\text {current }}, T_{\text {publish }}+T_{\text {all }}\right)$ represents the amount of information that is being scored at the current time, Count $\left(T_{\text {all }}\right)$ represents the amount of information that is being scored throughout the life cycle.

The influence of the information formula can also be expressed as a formula (2) for information follows a negative exponential model [10].

$$
\operatorname{Influence}\left(T_{\text {current }}, T_{\text {publish }}\right)=e^{-\alpha\left(T_{\text {current }}-T_{\text {publish }}\right)}
$$

Where, $T_{\text {current }}$ represents the current time, $T_{\text {publish }}$ represents the time of release of information, Influence $\left(T_{\text {current }}, T_{\text {publish }}\right)$ represents the size of the information at the current time, $\alpha$ represents aging coefficient of information.

The formula for calculating the aging coefficient of information given by [1] is shown in formula (3).

$$
\alpha=-\ln (0.5) / \bar{T}_{\text {half }}
$$

Where, $\bar{T}_{\text {half }}$ represents the average of the half life of all the information. According to the definition, half life means the time when the number of comments on information decays to half. It is easy to calculate the half-life with the click-through, and then the aging coefficient of information can be calculated by formula (3). Finally the influence of information can be obtained by formula (2).

\section{3 GORITHM BASED ON INFORMATION AGING}

\subsection{Algorithm design thoughts}

In order to solve the problem where the social network cannot filter out date information, this paper construct a trust network with information influence to generate a highly efficient recommendation list for users by the information aging theory in second section.

\subsection{Basic concepts and properties of trust network}

Based on the literature [9], this paper gives the basic concepts and properties of the trust network as follows.

Definition 4 (node): In the trust network $G$, each user $u_{i}$ is a node. If there are $m$ users, then $i$ belongs to $[0, m]$. Definition 5 (trust degree): In the trust network $G$, quantification of the trust degree of node $u_{i}$ to node $u_{j}$ is the trust degree of node $u_{i}$ on the node $u_{j}$, defined as $\operatorname{Trust}\left(u_{i}, u_{j}\right)$.

Definition 6(trust network): The trust relationship among users can generate a trust network. The trust network can be represented as a directed weighted graph $G(V, E$, Trust $) . V$ represents nodes in a graph, $E$ represents relationships among vertices, Trust represents trust degree among nodes.

Definition 7(direct trust degree): In the trust network $G$, the direct trust degree of node $u_{j}$ to node $u_{i}$ is obtained according to the direct interaction between them, defined 
as $\operatorname{DTrust}\left(u_{i}, u_{j}\right)$. In this paper, 0 represents totally not trust, 1 represents complete trust.

Definition 8(recommendation trust): In the trust network $G$, the recommendation trust degree of node $u_{j}$ to node $u_{i}$ refers to the trust degree calculated by the direct trust degree of the neighbor nodes of node $u_{i}$ to node $u_{i}$. It is also known as indirect trust, defined as $\operatorname{RTrust}\left(u_{i}, u_{j}\right)$.

Property 1(time sensitivity): In social networks, the trust relationship among people is constantly changing for the trust degree between friends will continue to decline because of the long-term noncontact. If the trust of $u_{i}$ to $u_{j}$ is $\operatorname{Trust}_{t_{1}}\left(u_{i}, u_{j}\right)$ at $t_{1}$, the trust of $u_{i}$ to $u_{j}$ is Trust $_{t_{2}}\left(u_{i}, u_{j}\right)$ at $t_{2}$ and $t_{1}<t_{2}$, then Trust $_{t_{1}}\left(u_{i}, u_{j}\right)<$ Trust $_{t_{2}}\left(u_{i}, u_{j}\right)$.

Property 2(asymmetry property): In the real world, the trust relationship among people is asymmetrical. Such as Tom is a friend of Jane. Tom trusts Jane, but Jane may not trust Tom. If the trust of Tom to Jane is Trust (Tom, Jane) and the trust of Jane to Tom is Trust (Jane,Tom) then Trust $($ Tom, Jane $)=\operatorname{Trust}($ Jane, Tom $)$ may be not valid.

Property 3(transitive property): In social network, the trust relationship among people is transitive. Such as Tom trusts Jane, and the trust degree is Trust (Tom, Jane). Jane trusts Mike, and the trust degree is Trust(Jane, Mike). Then Tom has indirect trust relationship with Mike and indirect trust of Tom to Mike

Trust $($ Tom, Mike $) \leq$ $\max \{$ Trust (Tom, Jane), Trust (Jane, Mike) $\}$

\subsection{Building data model}

(a)User model $U=\left\{u_{1}, u_{2}, u_{3} \ldots, u_{m}\right\}$ represents collection of users.

(b)Information model $I=\left\{i_{1}, i_{2}, i_{3} \ldots, i_{n}\right\}$ represents collection of information.

(c)The user rating matrix is a two-dimensional matrix composed of the rating of users $U$ on information $I$, defined as $R_{m \times n}$.

(d)The user direct trust matrix is a two-dimensional matrix composed of direct trust relationships among users U, defined as DTrust $t_{m \times m}$.

\subsection{Neighbor selection improved method}

(a) Calculation of direct trust degree

The direct trust between users is 0 or 1 in the dataset. This paper uses the direct trust value to measure the direct trust degree.

(b) Calculation of recommendation trust degree

By definition 8, recommendation trust degree refers to the trust degree of adjacent nodes (recommended nodes) to the target nodes in the trust network. The evaluation preference of recommendation nodes is different from that of the source nodes. For example, recommendation nodes are very satisfied with the evaluation of information, but the source nodes are not satisfied with the evaluation of the information. It reflects the individual differences between the evaluation information of the network nodes. Then the definition of similarity is introduced in the calculation of recommendation algorithm where the nodes will be given a high priority if they are similar to the source nodes.

Definition 9(evaluation similarity): In the trust network $G$, the similarity of node $u_{i}$ and $u_{j}$ is evaluation similarity, defined as $\Delta\left(u_{i}, u_{j}\right)$ 。

In this paper, the similarity is calculated by the PPC (correlation coefficient Pearson). The specific calculation formula is shown in formula (4).

$\Delta_{s i}= \begin{cases}\sum_{n \in N_{s i}}\left(R_{s n}-\bar{R}_{s}\right) \times\left(R_{i n}-\bar{R}_{i}\right) / H & \left|N_{s i}\right|>0 \text { and } H \neq 0 \\ 0 & \left|N_{s i}\right|=0 \text { or } H=0\end{cases}$

Where, $\quad H=\sqrt{\sum_{n \in N_{s i}}\left(R_{s n}-\bar{R}_{s}\right)^{2}} \times \sqrt{\sum_{n \in N_{s i}}\left(R_{i n}-\bar{R}_{i}\right)^{2}}, \quad N_{s i}$ represents a set of information that has been evaluated jointly by node $s$ and node $i, R_{s n}$ represents the score of $s$ on $n, \bar{R}_{s}$ represents the arithmetic mean of scores of node $s$ to all information in the dataset, $R_{\text {in }}$

represents the score of $i$ on $n, \bar{R}_{i}$ represents the arithmetic mean of scores of node $i$ to all information in the dataset.

According to the formula (4), $\Delta \in[-1,1], \Delta=1$ represents a complete positive correlation, $\Delta=-1$ represents a completely negative correlation, $\Delta=0$ represents is completely unrelated. $N=0$ represents there is no common evaluation information, and the similarity between node $S$ and node $i$ cannot be calculated at this moment, $\Delta=0 . N=1$ represents there is a common score information among nodes. The arithmetic mean of each node is the score information of the node at this moment, and it is unable to calculate the 
similarity by the formula (4). So the formula (4) is revised as formula (5).

$\Delta_{s i}= \begin{cases}\sum_{n \in N_{s i}}\left(R_{s n}-\bar{R}_{s}\right) \times\left(R_{i n}-\bar{R}_{i}\right) / H & \left|N_{s i}\right|>0 \text { and } H \neq 0 \\ 0 & \left|N_{s i}\right|=0 \text { or } H=0 \\ \left(\left|R_{s}-R_{i}\right|\right) /\left(R_{\max }-R_{\min }\right) & \left|N_{s i}\right|=1 \text { and } H \neq 0\end{cases}$

Where, $R_{\max }$ represents the maximum value in the set $N_{s i}, R_{\text {min }}$ represents the minimum value in the set $N_{s i}$.

$N^{\prime}$ is the set of nodes whose similarity between the source node is not zero. At the same time, the path length between recommended nodes and target nodes is no more than $L_{\max }$. That is $N^{\prime}=\left\{n \in N \mid \Delta_{s n}>0, L_{n t}<L_{\max }\right\} \quad, \quad$ where, $s$ represents source node, $t$ represents target node.

Based on the above analysis, the recommendation trust is defined as formula (6).

$$
\operatorname{RTrust}(s, t)= \begin{cases}\sum_{i \in N^{\prime}} \Delta_{s i} \operatorname{DTrust}(s, i) \Delta_{i t} \hat{C}(i, t) & N^{\prime} \neq \Phi \\ 0 & N^{\prime}=\Phi\end{cases}
$$

In the formula (6), if there are many paths between the recommended nodes and the target nodes is less than $L_{\text {max }}$, then $\hat{C}(i, t)=\max \left\{1 / L_{i}, i \in N\right\}$. If there is no path, then $\hat{C}(i, t)=0$.

\section{(c) Integration of trust degree}

In order to integrate the direct trust and recommendation trust, this paper introduces the trust regulatory factor [9], and the specific integration formula is shown in formula (7).

$$
\operatorname{Trust}(s, t)=\lambda D \operatorname{Trust}(s, t)+(1-\lambda) \operatorname{RTrust}(s, t)
$$

Where, the range of $\lambda$ is $(0,1)$. When $\lambda$ is bigger, the effect of direct trust degree on trust degree is greater, and the effect of recommendation trust degree on trust degree is smaller. When $\lambda$ is smaller, the effect of direct trust degree on trust degree is smaller, and the effect of recommendation trust degree on trust degree is greater. The value of $\lambda$ is mainly obtained by experience and cross experiment.

(d) Improvement of nearest neighbor selection

In order to take full account of the trust relationship among users and the timeliness of the information itself, this paper improved the traditional recommendation algorithm by integrating fusions trust and information influence. Fusion formula is shown in formula (8).

$$
\operatorname{ITrust}(s, t)=\operatorname{Trust}(s, t) \times \operatorname{Influence}\left(T_{\text {current }}, T_{\text {publish }}\right)
$$

Where, $\operatorname{ITrust}(s, t)$ represents the final trust degree between the user $s$ and $t$.

(e) Rating prediction

Based on the formula (8), this paper choose $U$ users who have maximum weight with the target users as the nearest neighbors set, then the rating of unknown item to current user can be predicted by the contribution of the nearest neighbors set. Concrete prediction formula is shown in the formula (9).

$$
R_{s i}=\bar{R}_{s}+\frac{\sum_{t \in U} \operatorname{ITrust}(s, t)\left(R_{t i}-\bar{R}_{t}\right)}{\sum_{t \in U} \operatorname{ITrust}(s, t)}
$$

\subsection{Recommended process description}

Input: User-item rating matrix, user-project trust matrix. Output: Users' recommendation information list.

Step 1: Evaluating the similarity among users. The evaluation similarity among the users is calculated by the formula (5) and the user's score matrix on the item.

Step 2: Calculating the path length between the recommended nodes and the destination nodes. Selecting the shortest path and calculating its reciprocal to get $\hat{C}(i, t)$.

Step 3: Computing recommendation trust among users. The recommendation trust degree among users is calculated by the formula (6), the evaluation similarity, the direct trust matrix and C. $\hat{C}(i, t)$.

Step 4: Computing trust degree among users. Based on the formula (8), the direct trust degree and recommendation trust degree are integrated to obtain the trust degree among users.

Step 5: Computing information influence. First the information aging coefficient is calculated by the formula (3), and then the user's influence is calculated by the formula (2).

Step 6: Choosing the nearest neighbors for the target user. The nearest neighbors of the target user are computed by the top-N nearest neighbor selection strategy and formula (8).

Step 7: Predicting target user rating for information which are not evaluated by target user. It is predicted by the target user's nearest neighbor and rating prediction formula (9).

Step 8: Generating recommendation list. According to the rating of target user's information, the information of the former top- $\mathrm{N}$ is selected as the recommended information for the target user. 


\section{EXPERIMENT}

\subsection{Data set and experimental environment}

This paper uses the epinions data set with timestamp to verify the timeliness of the proposed algorithm. The data set consists of two files. The epinion_trust_with_timestamp.mat file includes a trust relationship among 300548 users, each of which contains a time stamp when the trust is established. For example, $(1,2,3)$ represents the user 1 trusted users 2 in time 3. The file rating_with_timestamp.mat includes 922267 score information, each of which also contains time stamp of the rating. For example, $(1,2,3,4,5,6)$ represents user 1 has an evaluation record in time 6 for information 2. The specific rating is 5 points. Score ranges from 1 to 5 points. 3 represent the category of information. Data set contains 27 categories, this paper uses the data sets whose category is 3(movie). During the experiment, the user number is 1 to 1000 and all users are divided into two parts, $80 \%$ of the users are training data and the rest $20 \%$ is test data.

The value of trust regulation factor is 0.5 , which indicates that the function of the direct trust and recommendation trust among users are the same. The greater the biggest trust transfer level $L_{\max }$, the greater the degree of trust decay, the higher the complexity of the algorithm. Considering the complexity of the algorithm and the sparse degree of the trust matrix, it is easier to control the sparse degree of the trust matrix and the complexity of the algorithm when $L_{\max }=5$ is used.

Based on the above analysis, the variables in this algorithm are shown in Table 1.

Table 1 variable value table

\begin{tabular}{|c|c|c|}
\hline Name & Value & Remark \\
\hline$\lambda$ & 0.5 & Trust regulation factor \\
\hline $\mathrm{L}_{\max }$ & 5 & The maximum transfer level of trust \\
\hline
\end{tabular}

In the paper, the experimental environment is the Windows 7 operating system, 2GB memory, Intel (R) Core (TM) CPU i3-2330M @ 2.20GHz and the experimental programs are developed by matlab8.5.

In the paper, the experimental environment is the Windows 7 operating system, 2GB memory, Intel (R) Core (TM) CPU i3-2330M @ 2.20GHz and the experimental programs are developed by matlab8.5.

\subsection{Evaluating indicator}

The result of curve fitting is explained by mathematical statistics indicators, including R-square (coefficient of determination), adjusted R-square (degree of freedom adjusted R-square) and Sig value (significance). The closer to 1 for the value of R-square, the better the results of the curve fitting. The Sig value represents a significant test, which is divided into the equation significance test and the coefficient significance test, and the more close to 0 for its value, the more the equation and the coefficients are tested by the significance.

This paper uses the method of literature [1] to determine the level of timeliness and it is determined by the average timeliness of the recommended results. The specific formula is shown in the formula (10).

$$
\text { Timeliness }=\sum_{i \in I} \text { Influence }\left(T_{\text {current }}, T_{i, \text { publish }}\right) /|I|
$$

Where, Timeliness represens the average value of the timeliness of the recommendation list, $I$ represens recommendation list collection, $T_{\text {current }}$ represens current moment, $T_{i}$, publish represens the release time of information $i$, Influence $\left(T_{\text {current }}, T_{i, \text { publish }}\right)$ represens the influence of information $i$. Obviously, the larger the value of Timeliness, the higher the timeliness of the recommended results.

In this paper, the Mean (Absolute Error MAE) value is used to measure the accuracy of the prediction, which is defined as follows.

$$
M A E=\frac{\sum_{i=1}^{I}\left|p_{i}-q_{i}\right|}{|I|}
$$

Where, $p_{i}$ represens the predictive score of information $i, q_{i}$ represens the actual score of information $i, I$ represens recommendation collection.

\subsection{Experimental results and analysis}

(a) Verification experiment of negative exponential growth model

The rating data of 1000 users were processed and divided into 11 time periods according to the rating time. It is assumed that the release time is the earliest reviews time of the information. The number of reviews of information in each time period is counted to get the situation of information commented after the 11 time information is released. Specific data is shown in Table 2. The columns of the table indicate the released time and the rows of the table indicate the number of movies being commented at some time.

\begin{tabular}{|c|c|c|c|c|c|c|c|c|c|c|c|}
\hline \multirow{2}{*}{ Time } & \multicolumn{11}{|c|}{ Time range } \\
\hline & 1 & 2 & 3 & 4 & 5 & 6 & 7 & 8 & 9 & 10 & 11 \\
\hline 1 & 64807 & 0 & 0 & 0 & 0 & 0 & 0 & 0 & 0 & 0 & 0 \\
\hline 2 & 12365 & 10310 & 0 & 0 & 0 & 0 & 0 & 0 & 0 & 0 & 0 \\
\hline 3 & 3467 & 1955 & 5181 & 0 & 0 & 0 & 0 & 0 & 0 & 0 & 0 \\
\hline
\end{tabular}

Table 2 the number of movie review 


\begin{tabular}{rrrrrrrrrrrr}
\hline 4 & 2278 & 585 & 1571 & 5009 & 0 & 0 & 0 & 0 & 0 & 0 & 0 \\
5 & 2241 & 443 & 511 & 1334 & 5180 & 0 & 0 & 0 & 0 & 0 & 0 \\
6 & 1987 & 412 & 274 & 408 & 1184 & 4200 & 0 & 0 & 0 & 0 & 0 \\
7 & 1538 & 343 & 219 & 270 & 386 & 1049 & 4329 & 0 & 0 & 0 & 0 \\
8 & 1844 & 294 & 237 & 259 & 349 & 465 & 1124 & 4289 & 0 & 0 & 0 \\
9 & 2418 & 418 & 341 & 299 & 351 & 446 & 641 & 1694 & 4239 & 0 & 0 \\
10 & 1245 & 213 & 138 & 165 & 162 & 191 & 210 & 360 & 921 & 3166 & 0 \\
11 & 1453 & 245 & 147 & 120 & 146 & 163 & 184 & 210 & 355 & 693 & 3232 \\
\hline
\end{tabular}

In table 2, the number of comments of each time period is decreased with the increase of time, so it can be preliminarily determined that the change trend is consistent with the negative exponential growth model.
This experiment uses the data which is commented in time 1 and the data is normalized before the experiment. The results as shown in table 3 .

\begin{tabular}{lllllllllll}
\hline 1 & 2 & 3 & 4 & 5 & 6 & 7 & 8 & 9 & 10 & 11 \\
\hline 1 & 0.176 & 0.036 & 0.017 & 0.016 & 0.012 & 0.005 & 0.010 & 0.019 & 0.001 & 0.004 \\
\hline
\end{tabular}

The number of film reviews and time are fitted with shown in Figure 1, and the results of the parameters are exponential curve by SPSS in Table 3. The results are shown in Table 4.

Table 4 curve fitting result

\begin{tabular}{cccc}
\hline R-square & Adjusted R-square & Sig(equation) & Sig(coefficient) \\
\hline 0.718 & 0.687 & 0.001 & 0.001 \\
\hline
\end{tabular}

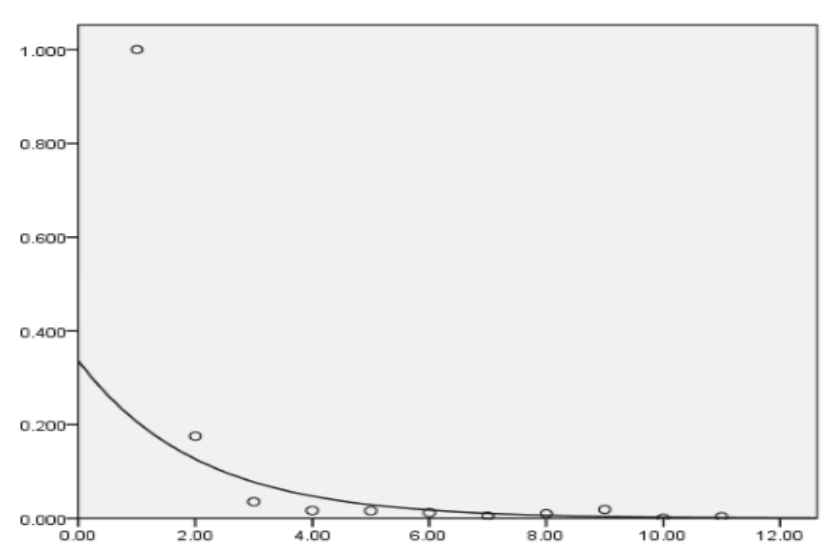

Fig. 1 curve fitting chart

R-square and Adjusted R-square are relatively large and Sig value approaches zero, equation and coefficients are tested by significance in Table 4 . The result shows that the fitting curve is consistent with the negative exponential growth model.

(b) Comparison experiment of algorithm timeliness The rating data and trust data of 1000 users are processed to get the user's trust matrix and the user's score matrix. The unknown rating of the target user is predicted by the similarity and trust between the nearest neighbors. Then the formula (10) is used to calculate the timeliness of the recommendation list. In order to reflect the high efficiency algorithm proposed in the paper, the experiment will analysis the result of our novel algorithm with the result of Cos-based collaborative filtering algorithm, person-based collaborative filtering algorithm and MoleTrust-based algorithm. The final timeliness is shown in Figure 2. The horizontal coordinates represent the number of recommended results and the longitudinal coordinates represent the timeliness of the recommended results.

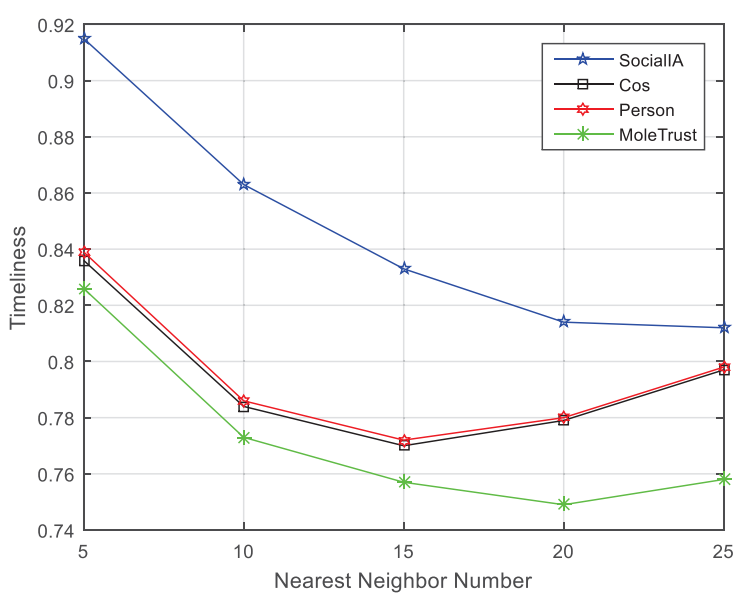

Fig. 2 Comparison of the timeliness of the algorithm As shown in Figure 2, the timeliness of the SociallA algorithm proposed in the paper is significantly higher than the other three algorithms. The main reason is that the SocialIA algorithm combines the trust degree of the users and the timeliness of the information itself when calculating the nearest neighbors. It significantly improves the timeliness of the recommended system and shields some overdue information.

(c) Comparative experiment of the influence of timeliness on the accuracy of the algorithm

In order to verify the timeliness of information in the proposed algorithm on the accuracy of the recommended list, the algorithm with timeliness and the algorithm without timeliness will be compared in the experiment. Comparison of the MAE values of the two algorithms is shown in figure 3 . 


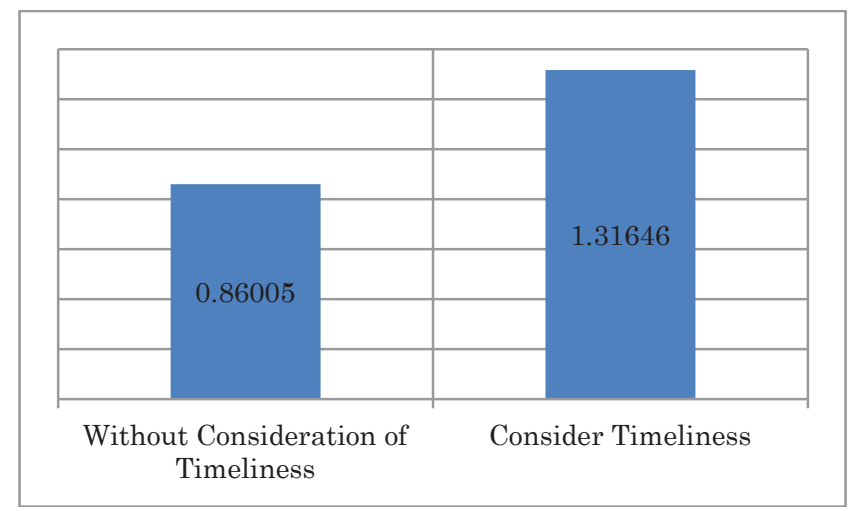

Fig. 3 Comparison of the MAE value of the algorithm

It is shown in Figure 3 that the algorithm with timeliness can reduce the accuracy of the algorithm. After the integration of trust degree and information influence in formula (8), the prediction rating of information has been increased, thus the MAE value of the recommended list becomes larger.

From the above analysis, we can know that the algorithm proposed in the paper can generate recommended list with high timeliness. The algorithm is suitable for recommendation systems with timeliness requirements, such as news recommendation, etc. But it will affect the accuracy of the final results. Therefore, this algorithm does not apply when the information itself is not sensitive to the timeliness or requires a higher accuracy.

\section{CONCLUSIONS}

In this paper, a negative exponential growth model is introduced to measure the influence of information for information aging problem. The experiment verifies that the number of the information and time on the public dataset meet the negative exponential growth model. At the same time, this paper also puts forward a kind of social network algorithm based on the theory of information aging. A new algorithm is proposed to improve the nearest neighbor selection rules by integrating the trust degree among users and the timeliness of information. Finally, the recommended list of high timeliness is generated for target user, and the timeliness of the algorithm is compared on the public dataset to verify its high timeliness. This algorithm does not apply when the information itself is not sensitive to the timeliness.

\section{Acknowledgements}

The authors would like to thank anonymous referees for their helpful comments and suggestions, and the Fundamental Research Funds for the Board of Education in Chongqing Municipality (KJ1403207) for support.

\section{REFERENCES:}

[1] Y. B. Wang, X. W. Meng, X. Hu, "Information Aging-based Collaborative FilteringRecommendationAlgorithm," J. of Electronics \& Information Technology, Vol. 35, No.10, pp. 2391-2396, 2013. (in Chinese)
[2] Bouadjenek M R, Hacid H, Bouzeghoub M, et al. PerSaDoR: Personalized Social Document Representation for Improving Web Search $\hbar[\mathrm{J}]$. Information Sciences, 2016, 369:614-633.

[3] Boroujeni F Z, Behnia M, Jahangard S. Improving collaborative recommendations using vector quantization and clustering $[\mathrm{J}]$. Social Network Analysis \& Mining, 2016, 6(1).

[4] P. Massa, P. Avesani, "Trust-aware recommender systems," Proceeding of ACM Conference on Recommender Systems, pp. 17-24, 2007.

[5] Nomatsu M, Suganuma Y, Yui Y. Development of an Autonomous Mobile Robot with Self-Localization and Searching Target in a Real Environment (Special Issue on Real World Robot Challenge in Tsukuba : Autonomous Technology for Useful Mobile Robot)[J]. Journal of Robotics \& Mechatronics, 2015, 27.

[6] M. Kukar, "Drifting Concepts as Hidden Factors in Clinical Studies,"M. of Artificial Intelligence in Medicine, Springer Berlin Heidelberg, pp. 28-35, 2003.

[7] Z Yang, Z Cai, S Liang, Y Zhang, "A Personalized Searching Algorithm Based on User's Interest Preferences and Semantic Similarity," J. of Computational Information Systems, Vol. 8, No. 6, pp.2501-2509, 2012.

[8] G. S. Yin, X. H. Cui, Z. Q. Ma, et al., "Forgetting curve-based collaborative filtering recommendation model," J. of Harbin Engineering University, 2012, 33(1): 85-90. (in Chinese)

[9] Z. B. Gan, C. Zeng, Y. Ma, et al., "C2C E-Commerce Trust Algorithm Based on Trust Network," J. of Software, Vol. 26, No. 8, pp.1946-1959, 2015. (in Chinese)

[10] H. K. Kim, H. Y. Oh, J. C. Gu, et al., "Commenders: A recommendation procedure for online book communities," J. OF Electronic Commerce Research \& Applications, Vol. 10, No. 5, pp.501-509, 2011. 